\title{
SULF2 is a novel diagnostic and prognostic marker for high-grade bladder cancer with lymphatic metastasis
}

\author{
Jianhua Huang ${ }^{1,2}$, Cheng Li $^{1,2}$, Wentao Zhang ${ }^{1,2}$, Fuhan Yang ${ }^{1,2}$, Ruiliang Wang ${ }^{1,2}$, Junfeng Zhang ${ }^{1,2}$, \\ Wei $\mathrm{Li}^{1,2}$, Xudong Yao ${ }^{1,2 \wedge}$ \\ ${ }^{1}$ Department of Urology, Shanghai Tenth People's Hospital, School of Medicine, Tongji University, Shanghai, China; ${ }^{2}$ Institute of Urinary Oncology, \\ School of Medicine, Tongji University, Shanghai, China \\ Contributions: (I) Conception and design: X Yao, W Li; (II) Administrative support: J Zhang; (III) Provision of study materials or patients: J Huang; (IV) \\ Collection and assembly of data: C Li, W Zhang; (V) Data analysis and interpretation: F Yang, R Wang; (VI) Manuscript writing: All authors; (VII) \\ Final approval of manuscript: All authors. \\ Correspondence to: Prof. Xudong Yao; Prof. Wei Li; Prof. Junfeng Zhang. Department of Urology, Shanghai Tenth People's Hospital, Tongji \\ University, Shanghai 200072, China. Email: yaoxudong1967@163.com; liweitongi@@163.com; 1610655@tongji.edu.cn.
}

Background: Sulfatase 2 (SULF2) is a member of the sulfatase family, and its expression and clinical significance in bladder cancer (BCa) are not currently known. In this study, we attempted to evaluate SULF2 expression in $\mathrm{BCa}$ patients who underwent radical cystectomy (RC) and the relationship between SULF2 expression and clinical-pathological characteristics.

Methods: Data on SULF2 expression in BCa tissues was obtained from the Oncomine database and the Gene Expression Omnibus (GEO). The expression of SULF2 and vascular endothelial growth factor-D (VEGF-D) in BCa was evaluated by immunohistochemistry (IHC) in tissues from 203 patients who had undergone RC. We also explored the value of the measurement of SULF2 and VEGF-D expression for diagnosis and prognosis in BCa patients with lymphatic metastasis.

Results: We found an increase in SULF2 messenger RNA (mRNA) levels and gene amplification in BCa tissues from the Oncomine database. High expression of SULF2 was detected in 91/203 (44.8\%) of BCa patients. Among these patients, 27 of 42 (64.3\%) with lymphatic metastasis showed high SULF2 expression. Univariate analysis showed that tumor size, pathological stage, lymphatic metastasis, vascular infiltration, perineural infiltration, hydronephrosis, and VEGF-D and SULF2 expression were related to prognosis in BCa patients, and multivariable Cox regression analysis showed that SULF2 expression was an independent prognostic indicator. Receiver operating characteristic (ROC) analysis revealed that SULF2 expression resulted in an increased area under the curve (AUC) of 0.707 , with a sensitivity of $71.4 \%$ and a specificity of $61.5 \%$.

Conclusions: The upregulation of SULF2 is associated with poor prognosis in high-grade BCa patients. It might be a novel diagnostic marker for BCa patients with lymphatic metastasis.

Keywords: Sulfatase 2 (SULF2); bladder cancer (BCa); lymphatic metastasis; diagnosis; prognosis

Submitted Jul 16, 2021. Accepted for publication Sep 09, 2021.

doi: $10.21037 / \mathrm{atm}-21-4102$

View this article at: https://dx.doi.org/10.21037/atm-21-4102

\footnotetext{
^ ORCID: 0000-0001-7234-3940.
} 


\section{Introduction}

Bladder cancer ( $\mathrm{BCa}$ ) is the most common malignant tumor of the urinary system and is the fourth most common malignant disease in males worldwide. Approximately $90 \%$ of pathology results indicate urothelial carcinoma. The majority of patients present with non-muscleinvasive bladder cancer (NMIBC), which can be treated with transurethral resection of bladder tumor (TURBT) and adjuvant administration of chemotherapy or Bacillus Calmette-Guerin (BCG) (1). Radical cystectomy (RC) with pelvic lymphadenectomy continues to be the gold standard for patients with muscle-invasive tumors and highrisk NMIBC (2). In patients with high-risk NMIBC and muscle-invasive bladder cancer (MIBC) undergoing RC, the accurate prediction of the status of lymph node (LN) metastasis could provide guidance for selecting patients who have an essential need for perioperative chemotherapy integrated with extended LN dissection at the time of RC.

Imaging examination via computed tomography (CT) or magnetic resonance imaging (MRI) is the primary tool used for evaluating preoperative LN metastasis at present, but it cannot provide an accurate patient assessment due to the limitations of imaging. Serological examination, urine examination, and histopathological examination also contribute important diagnostic information.

Sulfatase 2 (SULF2) is a member of the sulfatase family and plays an important role in cancer progression by changing the sulfate pattern of heparan sulfate proteoglycan (HSPG) located on the surface of most cancer cells (3). In tumor cells, the abnormal expression of SULF2 can lead to structural changes of proteoglycans, resulting in uncontrolled proliferation of tumor cells, increased invasion ability, and increased lymphatic metastasis. SULF2 is currently studied in a variety of tumors, but it is currently not studied in bladder cancer. In this study, we found that SULF2 is an important factor affecting the prognosis of bladder cancer and is closely related to tumor lymph node metastasis. At the same time, VEGF-D plays an important role in promoting lymphangiogenesis. In bladder cancer, it has also been reported that VEGF-D binds to vascular endothelial growth factor receptor 3 (VEGFR-3) to drive lymphangiogenesis and promote lymphatic metastasis. In this study, the ability of SULF2 and VEGF-D to predict lymphatic metastasis of bladder cancer will also be compared.

In our study, we retrospectively assessed $203 \mathrm{BCa}$ patients who had received RC and pelvic lymphadenectomy at Shanghai Tenth People's Hospital between 1 January 2013 and 31 December 2017. The aim of this study was to measure SULF2 expression in samples from the 203 patients and to determine whether SULF2 could serve as a novel diagnostic and prognostic marker for high-grade $\mathrm{BCa}$ patients with lymphatic metastasis.

We present the following article in accordance with the STARD reporting checklist (available at https://dx.doi. org/10.21037/atm-21-4102).

\section{Methods}

\section{Patients and tissue samples}

A total of 203 BCa patients who had received RC and pelvic lymphadenectomy at Shanghai Tenth People's Hospital, which is affiliated with Tongji University, between 1 January 2013 and 31 December 2017 were included. All tumors were graded histologically according to the World Health Organization (WHO) 2004 grading scheme and staged according to the 2002 Union of International Cancer Control (UICC) tumor, node, metastasis (TNM) classification system for BCa. The tissue samples were collected during surgery, and 2 independent experts used paraffin-fixed pathological specimens to confirm the diagnosis via histopathological examination. For all specimens, the clinical and pathological information, such as age, gender, pathology, differentiation, TNM stage, and follow-up data, was collected. Patients undergoing radiotherapy or chemotherapy before surgery were excluded from this study. The expression of SULF2 and vascular endothelial growth factor-D (VEGF-D) expression in $\mathrm{BCa}$ tissues was assessed through immunohistochemical (IHC) staining with specific antibodies. The study was approved by the Ethics Committee of the Shanghai Tenth People's Hospital (SHSY-IEC-4.1/19-120/01), and all participants provided written informed consent before registration. The study is also in line with the Helsinki Declaration (as revised in 2013).

\section{Analysis of GEO and Oncomine data}

The data of SULF2 expression were retrieved from the Gene Expression Omnibus (GEO, http://www.ncbi.nlm. nih.gov/geo) and the Oncomine database (www.oncomine. org). A total of 4 datasets were analyzed for SULF2 expression, including GSE13507, GSE23732, GSE27448, 
and GSE48075. The SULF2 copy number was assessed using messenger RNA (mRNA) expression data from the Dyrskjøt bladder studies, which was obtained from The Cancer Genome Atlas (TCGA) via the Oncomine database.

\section{IHC}

The specimens were obtained from normal bladder tissues adjacent to cancer tissues and embedded in paraffin after dehydration. A total of 4 consecutive $4 \mu \mathrm{m}$ thick paraffin sections were obtained from each participant. The sections were baked at $70{ }^{\circ} \mathrm{C}$ in a thermostat for $15 \mathrm{~min}$, dewaxed, and washed with phosphate-buffered saline (PBS) 3 times for 2 min each. The specimens were placed in an antigen repair box, immersed in $0.01 \mathrm{~mol}$ citric acid buffer, heated in a microwave oven for $15 \mathrm{~min}$, and cooled to room temperature. They were then rinsed in PBS 3 times for $2 \mathrm{~min}$ each time. The slices were incubated at room temperature for $15 \mathrm{~min}$ with $50 \mu \mathrm{L}$ peroxidase blocker and rinsed in PBS 3 times for 2 min each time. The PBS solution was removed, and the slices were incubated with goat serum at room temperature for $20 \mathrm{~min}$ in the dark. The excess serum was removed without washing. The slices were then incubated with $50 \mu \mathrm{L}$ primary antibody (SULF2 and VEGF-D, Abcam, Hangzhou, China). After washing in PBS 3 times, the sections were labeled with a second antibody for $1 \mathrm{~h}$ and again washed 3 times with PBS. The sections were developed with a 3,3'-diaminobenzidine (DAB) reagent kit and examined by microscopic observation and photography.

\section{Evaluation of IHC}

The SULF2-positive cells displayed brownish yellow granules in the cytoplasm and membrane. The evaluation of SULF2 staining included the intensity of staining (scored as 0 , no staining; 1 , weak staining; 2 , moderate staining; 3 , strong staining) and the percentage of positive tumor cells (scored as: $0,<5 \% ; 1,5-25 \% ; 2,26-50 \% ; 3,51-75 \% ; 4$, 76-100\%). To facilitate the statistical analysis, the SULF2 staining intensity and frequency were transformed into a composite expression score (CES) by utilizing the formula $\mathrm{CES}=$ intensity $\times$ frequency. The range of the CES was from 0 to 12. The CES was classified as follows: 0 , negative; $1-4$, weakly positive; $5-8$, positive; $9-12$, strongly positive.
These scores were independently assessed by 2 pathologists. Scores of 0-4 and 5-12 were indicative of low expression and high expression, respectively.

\section{Statistical analysis}

The software SPSS 19.0 (IBM Corp., Chicago, IL, USA) was used for statistical analysis. A chi-square $\left(\chi^{2}\right)$ test was used to analyze the relationships between the markers and clinicopathological features. Kaplan-Meier analysis was performed to evaluate the effects of SULF2 expression on the overall survival (OS) of patients with high-grade BCa. Cox regression was used for the analysis of risk factors. The biomarker levels were assessed through receiver operating characteristic (ROC) curves. A bilateral $\mathrm{P}<0.05$ was considered to indicate statistical significance.

\section{Results}

\section{SULF2 is upregulated in $B C a$}

We examined 2 datasets from Oncomine showing the expression of SULF2 in $\mathrm{BCa}$, and the results indicated that the SULF2 gene copy number was increased in BCa samples compared to that in normal bladder samples according to TCGA (Figure 1A). In addition, the SULF2 mRNA level in bladder urothelial carcinoma samples was also increased in comparison to that in normal samples in the Lee Cell Line 2 dataset (Figure 1B). The results indicated that the expression of SULF2 was increased in bladder urothelial carcinoma.

\section{Clinicopathological characteristics of bladder urothelial cancer patients}

A total of 203 urothelial BCa patients who had received RC and pelvic lymphadenectomy at Shanghai Tenth People's Hospital at Tongii University between 1 January 2013 and 31 December 2017 were retrospectively assessed in this study. The participant ages ranged from 32 to 86 years, with an average of 67 years. A total of 175 participants were male, and the rest were female. A total of 42 patients were diagnosed with $\mathrm{BCa}$ with lymphatic metastasis. In addition, other characteristics were also assessed, including tumor size, pathological stage, vascular infiltration, perineural infiltration, hydronephrosis, smoking, distant metastasis, 

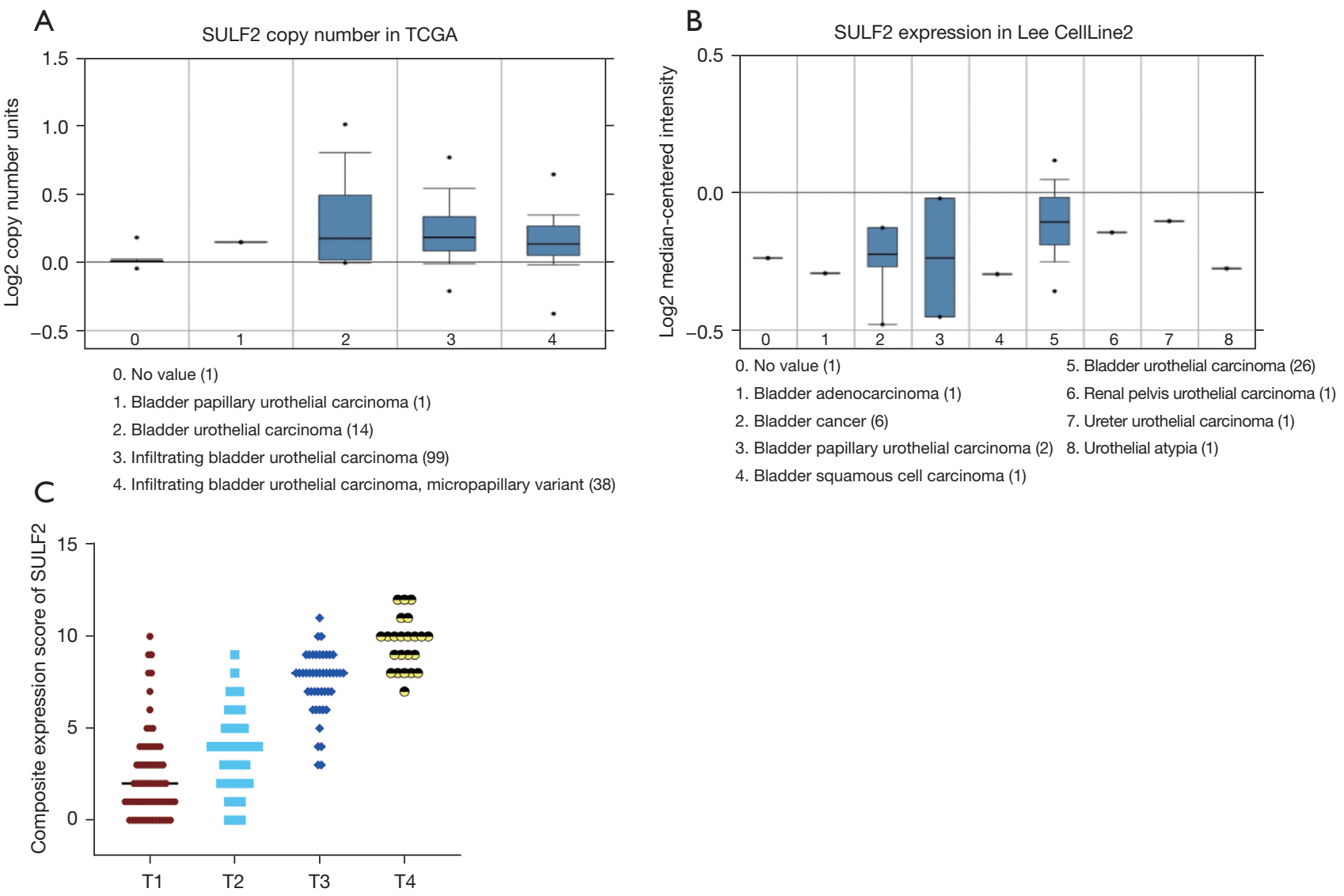

Figure 1 SULF2 DNA and mRNA expression in human BCa according to data from the Oncomine database. (A) SULF2 gene copy number in human BCa vs. that in normal bladder tissues. (B) SULF2 mRNA expression in human BCa vs. that in normal bladder tissues. (C) IHC score of SULF2 in 203 cases of BCa. BCa, bladder cancer; mRNA, messenger RNA; SULF2, sulfatase 2.

hypertension, diabetes, VEGF-D and SULF2 expression.

\section{Univariate analysis and multivariable Cox regression analysis of the association with prognosis in high grade BCa patients}

The clinicopathological characteristics of 203 patients with high-grade $\mathrm{BCa}$ who underwent $\mathrm{RC}$ with pelvic lymphadenectomy were retrospectively assessed. Univariate analysis and multivariable Cox regression analyses were performed, and the univariate analysis showed that tumor size, pathological stage, vascular infiltration, perineural infiltration, hydronephrosis, distant metastasis, lymphatic metastasis, VEGF-D, and SULF2 expression were prognostic factors $(\mathrm{P}<0.05)$ (Table 1). Further multivariable Cox regression analysis revealed that SULF2 expression and lymphatic metastasis were independent prognostic factors for high-grade $\mathrm{BCa}(\mathrm{P}<0.05)$ (Table 2).

\section{Measurement of SULF2 expression in BCa samples by IHC}

To reveal the clinical significance of SULF2 in high-grade $\mathrm{BCa}$, we assessed its expression and distribution in $\mathrm{BCa}$ samples by IH. We analyzed the expression of SULF2 in the T1-T4 stages and found that as the stage increased, the expression of SULF2 also gradually increased (Figure 1C). The results showed that 30 of 42 cases with lymphatic metastasis exhibited high expression of SULF2, while 62 of 161 cases without lymphatic metastasis exhibited high expression of SULF2 (Figure 2A-2C), and the difference was statistically significant $(\mathrm{P}=0.004)$; thus, $\mathrm{SULF} 2 \mathrm{might}$ be associated with lymphatic metastasis in BCa. Then, we detected lymphangiogenesis-related factor VEGF-D (Figure 2D,2E), a member of the VEGF family, which showed high expression in 28 of 42 patients with lymphatic metastasis and 68 of 161 patients without lymphatic 
Table 1 Univariate analysis of $\mathrm{BCa}$

\begin{tabular}{lcc}
\hline Clinicopathological parameters & Hazard ratio & P value \\
\hline Age $(>60 / \leq 60)$ & 1.2110 & 0.4469 \\
Gender (male/female) & 1.3700 & 0.4575 \\
Tumor size (>3 cm/ $\leq 3 \mathrm{~cm})$ & 1.8770 & 0.0100 \\
Pathological stage (T1/T2-T4) & 2.8550 & 0.0004 \\
Lymphatic metastasis (+/-) & 5.0090 & $<0.0001$ \\
Vascular infiltration (+/-) & 2.0090 & 0.0047 \\
Perineural infiltration (+/-) & 2.4940 & 0.0016 \\
Hydronephrosis (yes/no) & 2.0980 & 0.0052 \\
Distant metastasis (yes/no) & 4.3450 & $<0.0001$ \\
Smoking (yes/no) & 1.1310 & 0.6259 \\
Hypertension (yes/no) & 1.2440 & 0.3825 \\
Diabetes (yes/no) & 0.9579 & 0.8956 \\
SULF2 expression (high/low) & 2.7151 & $<0.0001$ \\
VEGF-D expression (high/low) & 1.7650 & 0.0225 \\
\hline
\end{tabular}

BCa, bladder cancer; SULF2, sulfatase 2; VEGF-D, vascular endothelial growth factor-D.

Table 2 Multivariable Cox regression analysis of $\mathrm{BCa}$

\begin{tabular}{lcc}
\hline Clinicopathological parameters & Hazard ratio & P value \\
\hline Tumor size $(>3 \mathrm{~cm} / \leq 3 \mathrm{~cm})$ & 0.835 & 0.514 \\
Pathological stage (T1/T2-T4) & 0.710 & 0.429 \\
Lymphatic metastasis (+/-) & 4.255 & $<0.0001$ \\
Vascular infiltration (+/-) & 1.115 & 0.719 \\
Perineural infiltration (+/-) & 1.296 & 0.486 \\
Distant metastasis (yes/no) & 1.825 & 0.150 \\
SULF2 expression (high/low) & 2.217 & 0.028 \\
VEGF-D expression (high/low) & 1.751 & 0.100 \\
Hydronephrosis (yes/no) & 1.725 & 0.101 \\
\hline
\end{tabular}

BCa, bladder cancer; SULF2, sulfatase 2; VEGF-D, vascular endothelial growth factor-D.

metastasis, and the difference in expression was significant $(\mathrm{P}=0.005)$. In addition, statistical analysis showed that the expression levels of SULF2 were significantly correlated with pathological stage $(\mathrm{P}<0.001)$, distant metastasis $(\mathrm{P}<0.01)$, vascular infiltration $(\mathrm{P}=0.001)$, lymphatic metastasis $(\mathrm{P}=0.004)$, and VEGF-D expression. No statistically significant correlations were identified with other characteristics, including age, gender, tumor size and perineural infiltration (Table 3). Meanwhile, Pearson correlation analysis revealed that the expression level of SULF2 was closely associated with the expression of VEGF-D ( $\mathrm{r}=0.600, \mathrm{P}<0.001)$ (Table 4).

\section{High expression of SULF2 correlates with poor prognosis in bigh-grade BCa}

To investigate the relationship between SULF2 expression and clinical outcomes, we analyzed the prognostic significance of SULF2 and several clinicopathological characteristics using Kaplan-Meier analysis. The results showed that the OS time of participants with high expression of SULF2 was obviously decreased compared to that of those with low expression of SULF2 (Figure 3A). In addition, we discovered that patients with high VEGF-D expression had a poor prognosis (Figure 3B). Furthermore, patients with lymphatic metastasis, MIBC, vascular infiltration, perineural infiltration, hydronephrosis, and a tumor size of more than $3 \mathrm{~cm}$ had a poor prognosis (Figure $3 C-3 H$ ), while other clinicopathological characteristics, such as disease type, hypertension, diabetes, smoking, age, and gender, had no correlation with prognosis in the different groups (Figure 3I-3N).

\section{The diagnostic value of SULF2 in BCa with lymphatic metastasis}

To further assess the utility of SULF2 as a biomarker in the diagnosis of lymphatic metastasis in BCa patients alone or in combination with other indicators, we applied ROC curve analysis to assess SULF2 detection and CT or MRI in the diagnosis of $\mathrm{BCa}$ patients with lymphatic metastasis. Furthermore, we compared SULF2 detection and image examination for the pathological diagnosis of BCa. The results showed that the sensitivity and specificity of SULF2 expression were $71.4 \%$ and $61.5 \%$ for the diagnosis of $\mathrm{BCa}$ with lymphatic metastasis, respectively, while the sensitivity and specificity of image examination were $26.2 \%$ and $89.4 \%$, respectively (Tables 5,6). The AUCs for SULF2 detection, image examination, and the combination of SULF2 and image examination were 0.707 [95\% confidence interval (CI): 0.609 to 0.806 ], 0.578 (95\% CI: 0.476 to 0.681 ), and 0.725 (95\% CI: 0.629 to 0.821 ), respectively (Figure $4 A-4 C$ ). 

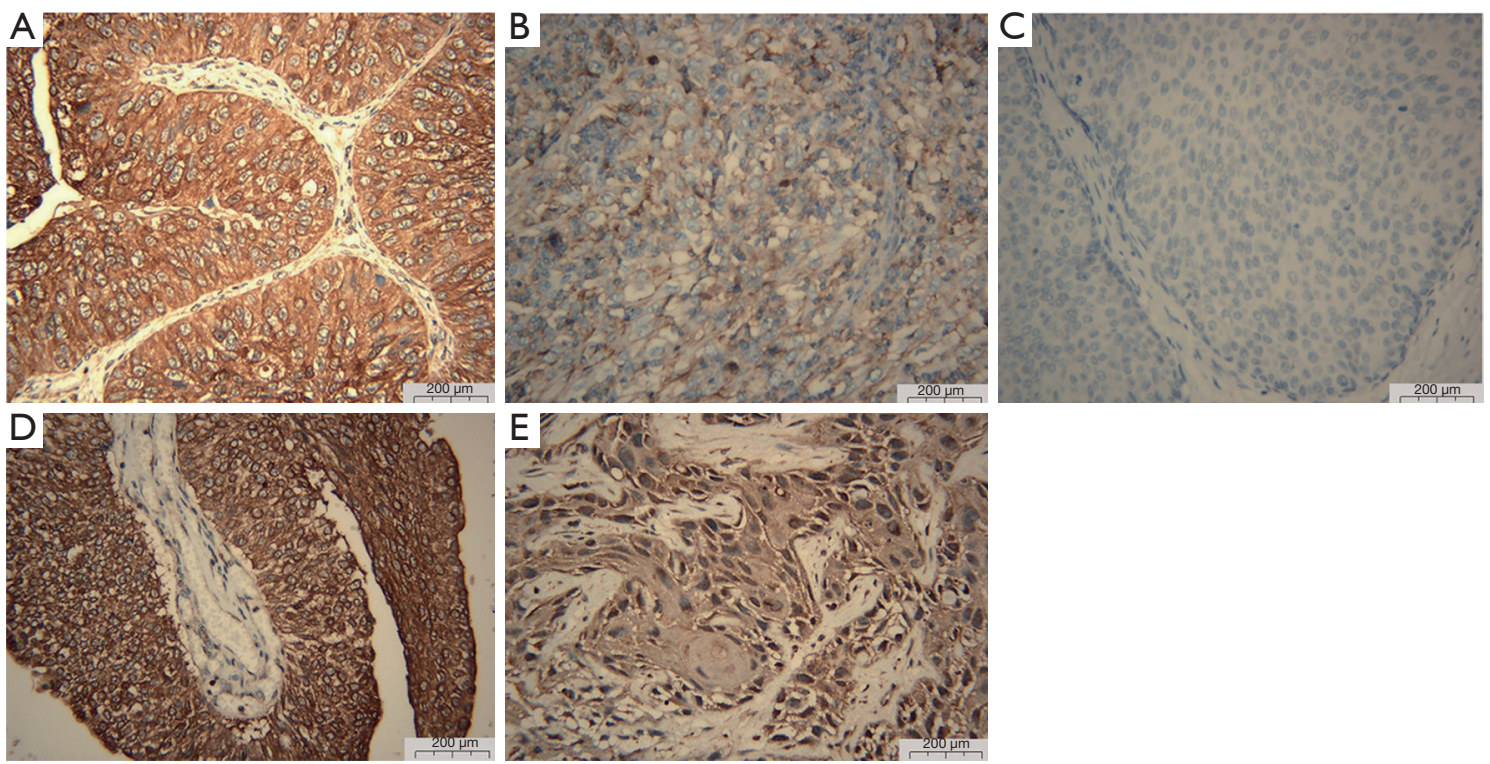

Figure 2 SULF2 and VEGF-D expression in human BCa measured by immunohistochemistry. (A) High expression of SULF2 (400×). (B) Low expression of SULF2 (400x). (C) Negative expression of SULF2 (400x). (D) High expression of VEGFD (400x). (E) Low expression of VEGFD (400×). BCa, bladder cancer; SULF2, sulfatase 2; VEGF-D, vascular endothelial growth factor-D.

Table 3 Correlation between SULF2 expression and clinicopathological characteristics in 203 patients with $\mathrm{BCa}$

\begin{tabular}{|c|c|c|c|c|}
\hline \multirow{2}{*}{$\begin{array}{l}\text { Clinicopathological } \\
\text { parameters }\end{array}$} & \multicolumn{3}{|c|}{ SULF2 expression } & \multirow{2}{*}{$P$ value } \\
\hline & Number & High & Low & \\
\hline Age & & & & 0.422 \\
\hline$\leq 65$ & 93 & 45 & 48 & \\
\hline$>65$ & 110 & 47 & 63 & \\
\hline Gender & & & & 0.900 \\
\hline Male & 175 & 79 & 96 & \\
\hline Female & 28 & 13 & 15 & \\
\hline Tumor size & & & & 0.422 \\
\hline$\leq 3 \mathrm{~cm}$ & 110 & 47 & 63 & \\
\hline$>3 \mathrm{~cm}$ & 93 & 45 & 48 & \\
\hline Pathological stage & & & & $<0.001$ \\
\hline $\mathrm{T} 1$ & 73 & 19 & 54 & \\
\hline T2-T4 & 130 & 73 & 57 & \\
\hline Distant metastasis & & & & $<0.01$ \\
\hline MO & 188 & 80 & 108 & \\
\hline M1 & 15 & 12 & 3 & \\
\hline
\end{tabular}

Table 3 (continued)
Table 3 (continued)

\begin{tabular}{|c|c|c|c|c|}
\hline \multirow{2}{*}{$\begin{array}{l}\text { Clinicopathological } \\
\text { parameters }\end{array}$} & \multicolumn{3}{|c|}{ SULF2 expression } & \multirow{2}{*}{$-P$ value } \\
\hline & Number & High & Low & \\
\hline Vascular infiltration & & & & 0.001 \\
\hline Yes & 63 & 39 & 24 & \\
\hline No & 140 & 53 & 87 & \\
\hline Perineural infiltration & & & & 0.184 \\
\hline Yes & 27 & 15 & 12 & \\
\hline No & 176 & 77 & 99 & \\
\hline Smoking & & & & 0.114 \\
\hline Yes & 116 & 47 & 69 & \\
\hline No & 87 & 45 & 42 & \\
\hline Lymphatic metastasis & & & & 0.004 \\
\hline Yes & 42 & 30 & 12 & \\
\hline No & 161 & 62 & 99 & \\
\hline VEGF-D expression & & & & $<0.001$ \\
\hline High & 98 & 34 & 64 & \\
\hline Low & 105 & 58 & 47 & \\
\hline
\end{tabular}


Table 4 Correlations between the expression of SULF2 and VEGF-D

\begin{tabular}{llc}
\hline & & VEGF-D \\
\hline SULF2 & Pearson correlation & $0.600^{* *}$ \\
& Sig. (double-tailed) & $<0.001$ \\
& $\mathrm{~N}$ & 203 \\
\hline
\end{tabular}

**, correlation is significant at the 0.01 level (double-tailed). SULF2, sulfatase 2; VEGF-D, vascular endothelial growth factor-D.

\section{Discussion}

In China, $\mathrm{BCa}$ is the most common urinary malignant tumor and a cause of significant burden in terms of morbidity and mortality (4). Pelvic lymphatic metastasis is the main form of metastasis and is also an important factor associated with pathological stage, treatment selection, the extent of LN dissection, and prognosis (5). We retrospectively assessed 203 BCa patients who had received $\mathrm{RC}$ and pelvic lymphadenectomy at the Shanghai Tenth People's Hospital at Tongji University between 1 January 2013 and 31 December 2017, and 20.7\% (42/203) of these patients were diagnosed with $\mathrm{BCa}$ with lymphatic metastasis. Wang et al. (6) reported that the percentage of lymphatic metastasis in $\mathrm{BCa}$ patients was $25 \%$. Lymphatic metastasis is the major cause of death in BCa patients. In our study, through univariate analysis, we found that tumor size, pathological stage, vascular infiltration, perineural infiltration, hydronephrosis, distant metastasis, lymphatic metastasis, and VEGF-D and SULF2 expression were prognostic factors. In addition, multivariable Cox regression analysis showed that SULF2 expression and lymphatic metastasis were independent prognostic factors for $\mathrm{BCa}$. The $\mathrm{LN}$ status is a powerful predictor of cancer-specific survival and a significant determinant of the selection of the therapeutic used following surgery $(7,8)$. Therefore, we believe that lymphatic metastasis is a critical factor for the prognosis of $\mathrm{BCa}$ patients.

RC combined with bilateral lymphadenectomy lymph node dissection (LND) is the gold standard treatment for patients with high-risk NMIBC and MIBC (9). The importance of lymphadenectomy during $\mathrm{RC}$ is generally accepted (10), but no consensus exists regarding the optimal extent of lymphadenectomy, the number of LNs to be retrieved, or the magnitude of the therapeutic benefit in patients with BC undergoing RC. Vieweg et al. (11) reported that only a limited number of patients with pelvic LN metastases who underwent extended LND showed benefit in terms of recurrence-free survival, while many studies have demonstrated improved survival in patients treated with extended bilateral LND compared to those who underwent standard or limited LND $(12,13)$. The method used to estimate the preoperative LN status is crucial for BCa patients.

The LN staging should be performed in patients before RC using imaging techniques such as MRI or enhanced CT scan (14), but the discrepancy between preoperative staging and the postoperative histopathological result is obvious (15). Many academics have started to explore other methods to assess LN status, such as serological examination, urine examination, and histopathological examination $(16,17)$. In this study, we retrospectively analyzed SULF2 expression in 203 BCa patients and found a discrepancy in SULF2 expression between patients with lymphatic metastasis and those without lymphatic metastasis, and we also showed that SULF2 expression is a prognostic and diagnostic marker for BCa. VEGF-D is closely related to tumor lymphangiogenesis and lymphatic metastasis. The expression of VEGF-D in tumor cells can not only induce the formation of lymph vessels in tumor tissues, but also promote the spread of tumor cells to lymph nodes. Through the analysis of the clinical data and prognosis of patients, we found that SULF2 can better predict lymphatic metastasis than VEGF-D, but the specific mechanism may still need to be further explored.

A member of the sulfatase family, SULF2, plays an important role in cancer progression by modifying the sulfate patterns in HSPGs located on the surfaces of most cancer cells (3). Zhu et al. (18) confirmed that SULF2 promoted lymphangiogenesis in breast cancer, and SULF2 was also upregulated in hepatocellular carcinoma, lung cancer, and pancreatic carcinoma (19-21). In this study, we investigated SULF2 expression in high-grade BCa through IHC, and 45.3\% (92/203) of samples showed high SULF2 expression. Moreover, 71.4\% (30/42) of samples showed high SULF2 expression in $42 \mathrm{BCa}$ patients with lymphatic metastasis, and the difference was significant. Therefore, we predicted that SULF2 might participate in the progression of lymphatic metastasis in BCa. In addition, we detected the expression of VEGF-D, a member of the vascular endothelial growth factor family, which has been shown to be related to lymphangiogenesis and lymphatic metastasis in malignant tumors (22). In this study, we found that the 
A
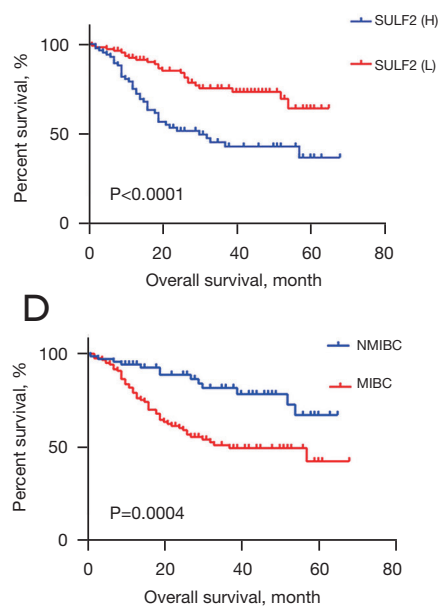

G
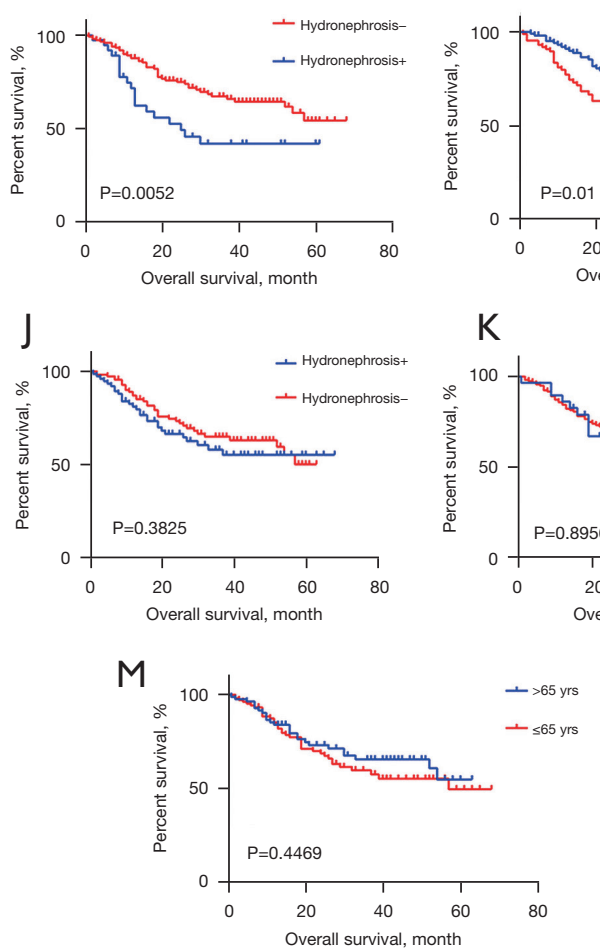

B

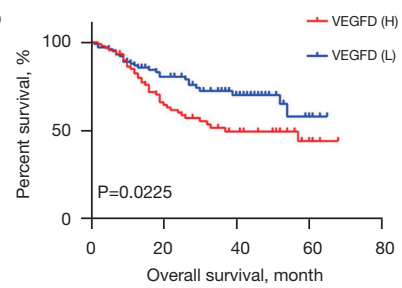

E
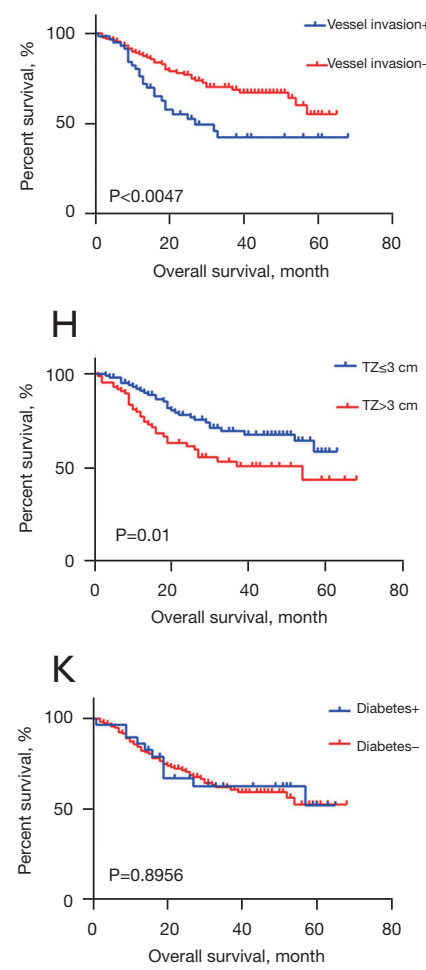

C

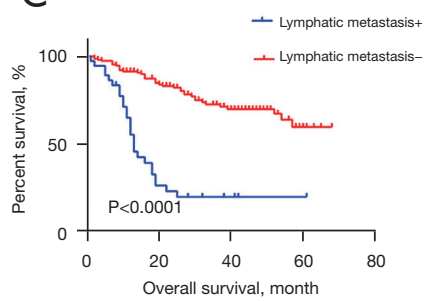

$\mathrm{F}$
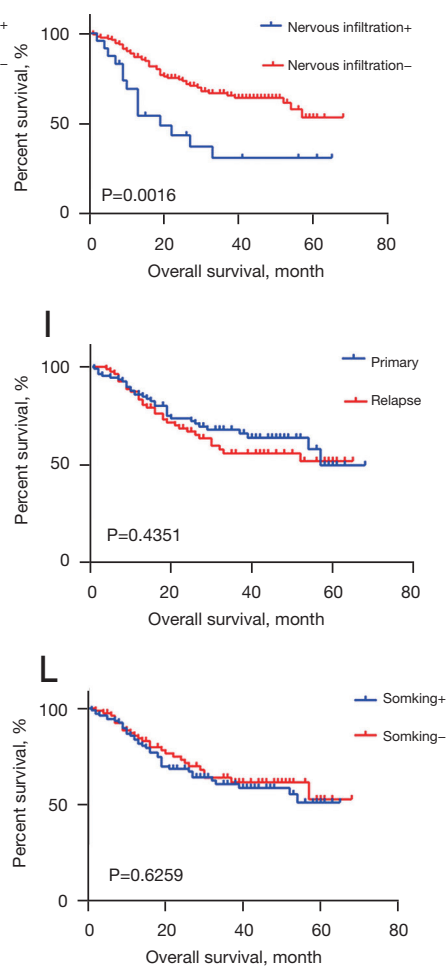

Figure 3 Survival curves for BCa generated using the Kaplan-Meier method and the log-rank test. (A) OS curves for patients with high SULF2 expression (blue line) and patients with low SULF2 expression (red line) $(\mathrm{P}<0.05)$; (B) OS curves for patients with high VEGF-D expression (blue line) and patients with low VEGF-D expression (red line) $(\mathrm{P}<0.05)$; (C) OS curves for patients with lymphatic metastasis (blue line) and patients without lymphatic metastasis (red line) ( $\mathrm{P}<0.05)$; (D) OS curves for patients with NMIBC (blue line) and patients with MIBC (red line) $(\mathrm{P}<0.05)$; (E) OS curves for patients with vessel infiltration (blue line) and patients without vessel infiltration (red line) $(\mathrm{P}<0.05)$; (F) OS curves for patients with perineural infiltration (blue line) and patients without perineural infiltration (red line) $(\mathrm{P}<0.05) ;(\mathrm{G})$ OS curves for patients with hydronephrosis (blue line) and patients without hydronephrosis (red line) $(\mathrm{P}<0.05)$; $(\mathrm{H})$ OS curves for patients with different tumor sizes $(\mathrm{P}<0.05)$; (I-N) OS curves for patients associated with disease type, hypertension, diabetes, smoking, age, and gender ( $\mathrm{P}>0.05)$. BCa, bladder cancer; SULF2, sulfatase 2; VEGF-D, vascular endothelial growth factor-D; OS, overall survival; NIMBC, non-muscle-invasive bladder cancer; MIBC, muscle-invasive bladder cancer. 
expression of SULF2 was closely associated with VEGF-D expression, so we hypothesized that SULF2 might promote LN metastasis through the VEGF-D pathway.

In this study, we revealed that SULF2 is a prognostic marker for $\mathrm{BCa}$ through univariate analysis and

Table 5 Imaging diagnosis of BCa with lymphatic metastasis

\begin{tabular}{lccc}
\hline \multirow{2}{*}{$\begin{array}{l}\text { Imaging } \\
\text { diagnosis }\end{array}$} & \multicolumn{2}{c}{ Pathological diagnosis } & \multirow{2}{*}{ Total } \\
\cline { 2 - 3 } & Positive $(+)$ & Negative $(-)$ & \\
\hline Positive (+) & $11(\mathrm{a})$ & $17(\mathrm{~b})$ & 28 \\
Negative $(-)$ & $31(\mathrm{c})$ & $144(\mathrm{~d})$ & 175 \\
Total & 42 & 161 & 203 \\
\hline
\end{tabular}

Sensitivity $26.2 \%[\mathrm{a} /(\mathrm{a}+\mathrm{c})]$; specificity $89.4 \%[\mathrm{~d} /(\mathrm{b}+\mathrm{d})]$; positive predictive value $39.3 \%[a /(a+b)]$; negative predictive value $82.3 \%[\mathrm{~d} /(\mathrm{c}+\mathrm{d})] . \mathrm{BCa}$, bladder cancer.

Table 6 Use of SULF2 expression for the diagnosis of BCa with lymphatic metastasis

\begin{tabular}{lccc}
\hline \multirow{2}{*}{$\begin{array}{l}\text { SULF2 } \\
\text { expression }\end{array}$} & \multicolumn{2}{c}{ Pathological diagnosis } & Total \\
\cline { 2 - 3 } & Positive (+) & Negative $(-)$ & \\
\hline Positive (+) & $30(\mathrm{a})$ & $62(\mathrm{~b})$ & 92 \\
Negative (-) & $12(\mathrm{c})$ & $99(\mathrm{~d})$ & 111 \\
Total & 42 & 161 & 203 \\
\hline
\end{tabular}

Sensitivity $71.4 \%[\mathrm{a} /(\mathrm{a}+\mathrm{c})]$; specificity $61.5 \%[\mathrm{~d} /(\mathrm{b}+\mathrm{d})]$; positive predictive value $32.6 \%[a /(a+b)]$; negative predictive value $89.2 \%[\mathrm{~d} /(\mathrm{c}+\mathrm{d})]$. BCa, bladder cancer; SULF2, sulfatase 2 . multivariable Cox regression analysis. Kaplan-Meier analysis showed that patients with high SULF2 expression had a shorter OS than those with low SULF2 expression. In practice, we can predict prognosis in $\mathrm{BCa}$ patients through the level of SULF2 expression.

Lymphatic metastasis is the most important factor associated with mortality in $\mathrm{BCa}$, so identifying the preoperative LN status is critical for early treatment. However, imaging techniques cannot provide specific information regarding preoperative LN status. In this study, the diagnosis rate when using imaging techniques for lymphatic metastasis was only $26.2 \%$, while the sensitivity of SULF2 expression when used for the diagnosis of lymphatic metastasis was $71.4 \%$. The difference was significant, so we speculate that the assessment of SULF2 expression may be more precise way to appraise the status of $\mathrm{LN}$ metastasis in BCa patients prior to surgery.

There are some limitations in this study. (I) This is a single-center study, and we hope that the next step will be a large-sample multi-center study. (II) The combined diagnostic efficiency of imaging and SULF2 may need to be verified for different machines. (III) The specific mechanism of SULF2 in lymphatic metastasis of bladder cancer needs further research and verification.

In conclusion, our study demonstrated that high SULF2 expression in $\mathrm{BCa}$ was associated with poor survival and that it provides a more precise method for the diagnosis for lymphatic metastasis. Therefore, we hypothesized that SULF2 is a novel diagnostic and prognostic marker for high-grade $\mathrm{BCa}$ with lymphatic metastasis.

\section{A}

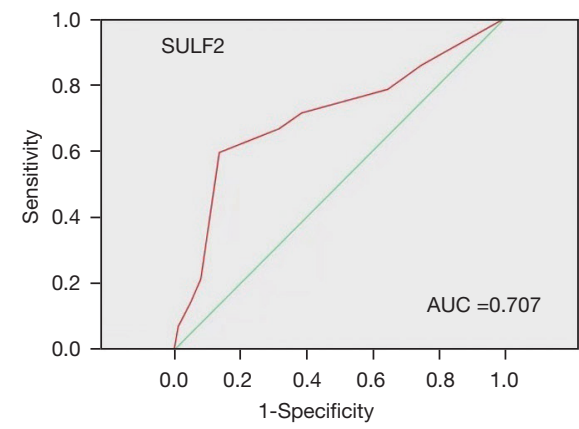

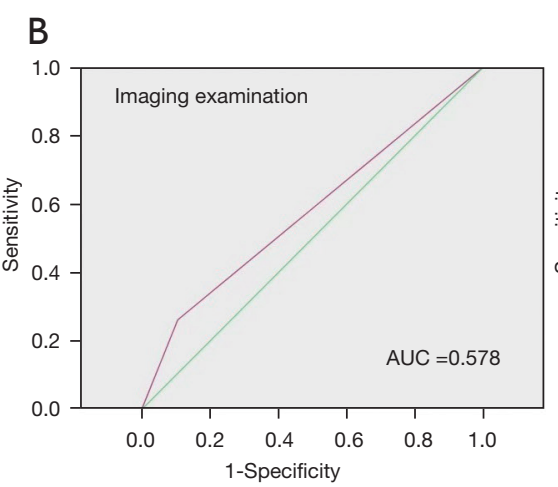

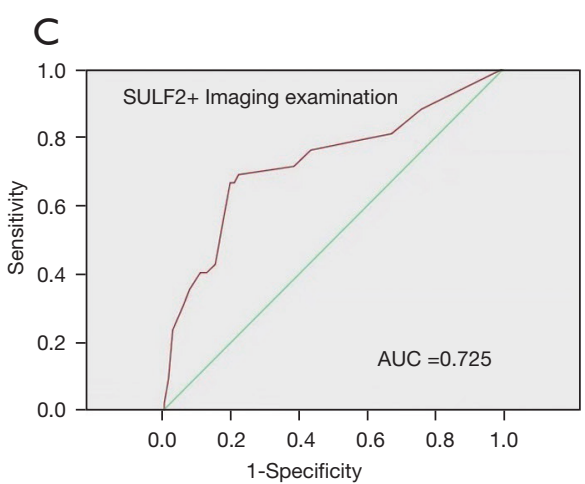

Figure 4 The diagnostic value of SULF2 in bladder urothelial carcinoma patients with lymphatic metastasis. (A) Receiver operator curves demonstrating the ability of SULF2 expression to predict bladder urothelial carcinoma with lymphatic metastasis. (B) Receiver operator curves demonstrating the ability of imaging examination to predict bladder urothelial carcinoma with lymphatic metastasis. (C) Receiver operator curves demonstrating the ability of a combination of SULF2 expression and imaging examination to predict bladder urothelial carcinoma with lymphatic metastasis. 


\section{Acknowledgments}

Funding: This work was supported in part by a grant from the National Natural Science Foundation of China (\#81472389); the Shanghai Science Committee Foundation (\#19411967700); National Natural Science Foundation of China, Youth Project (\#81602469); Shanghai Pujiang Talent Program (\#20PJ1412400) and General project of Shanghai Natural Science Foundation of China (\#20ZR1443000).

\section{Footnote}

Reporting Checklist: The authors have completed the STARD reporting checklist. Available at https://dx.doi. org/10.21037/atm-21-4102

Data Sharing Statement: Available at https://dx.doi. org/10.21037/atm-21-4102

Conflicts of Interest: All authors have completed the ICMJE uniform disclosure form (available at https://dx.doi. org/10.21037/atm-21-4102). The authors have no conflicts of interest to declare.

Ethical Statement: The authors are accountable for all aspects of the work in ensuring that questions related to the accuracy or integrity of any part of the work are appropriately investigated and resolved. The study was approved by the Ethics Committee of the Shanghai Tenth People's Hospital (SHSY-IEC-4.1/19-120/01), and all participants provided written informed consent before registration. The study is also in line with the Helsinki Declaration (as revised in 2013).

Open Access Statement: This is an Open Access article distributed in accordance with the Creative Commons Attribution-NonCommercial-NoDerivs 4.0 International License (CC BY-NC-ND 4.0), which permits the noncommercial replication and distribution of the article with the strict proviso that no changes or edits are made and the original work is properly cited (including links to both the formal publication through the relevant DOI and the license). See: https://creativecommons.org/licenses/by-nc-nd/4.0/.

\section{References}

1. Sörenby A, Baseckas G, Bendahl PO, et al. Reducing recurrence in non-muscle-invasive bladder cancer by systematically implementing guideline-based recommendations: effect of a prospective intervention in primary bladder cancer patients. Scand J Urol 2019;53:109-15.

2. Meeks JJ, Bellmunt J, Bochner BH, et al. A systematic review of neoadjuvant and adjuvant chemotherapy for muscle-invasive bladder cancer. Eur Urol 2012;62:523-33.

3. Maltseva I, Chan M, Kalus I, et al. The SULFs, extracellular sulfatases for heparan sulfate, promote the migration of corneal epithelial cells during wound repair. PLoS One 2013;8:e69642.

4. He YT, Li DJ, Liang D, et al. Incidence and mortality of bladder cancer in China, 2014. Zhonghua Zhong Liu Za Zhi 2018;40:647-52.

5. Moschini M, Mattei A, Cornelius J, et al. Surgical treatment for clinical node-positive bladder cancer patients treated with radical cystectomy without neoadjuvant chemotherapy. World J Urol 2018;36:639-44.

6. Wang L, Mudaliar K, Mehta V, et al. Seeking a standard for adequate pathologic lymph node staging in primary bladder carcinoma. Virchows Arch 2014;464:595-602.

7. VAN Bruwaene S, Costello AJ, VAN Poppel H. Prognosis of node-positive bladder cancer in 2016. Minerva Urol Nefrol 2016;68:125-37.

8. Rink M, Hansen J, Cha EK, et al. Outcomes and prognostic factors in patients with a single lymph node metastasis at time of radical cystectomy. BJU Int 2013;111:74-84.

9. Tilki D, Brausi M, Colombo R, et al. Lymphadenectomy for bladder cancer at the time of radical cystectomy. Eur Urol 2013;64:266-76.

10. Burkhard FC, Roth B, Zehnder P, et al. Lymphadenectomy for bladder cancer: indications and controversies. Urol Clin North Am 2011;38:397-405, v.

11. Vieweg J, Whitmore WF Jr, Herr HW, et al. The role of pelvic lymphadenectomy and radical cystectomy for lymph node positive bladder cancer. The Memorial Sloan-Kettering Cancer Center experience. Cancer 1994;73:3020-8.

12. Simone G, Papalia R, Ferriero M, et al. Stage-specific impact of extended versus standard pelvic lymph node dissection in radical cystectomy. Int J Urol 2013;20:390-7.

13. May M, Herrmann E, Bolenz C, et al. Association between the number of dissected lymph nodes during pelvic lymphadenectomy and cancer-specific survival in patients with lymph node-negative urothelial carcinoma of the bladder undergoing radical cystectomy. Ann Surg Oncol 
2011;18:2018-25.

14. Stenzl A, Cowan NC, De Santis M, et al. Update of the Clinical Guidelines of the European Association of Urology on muscle-invasive and metastatic bladder carcinoma. Actas Urol Esp 2010;34:51-62.

15. Shariat SF, Palapattu GS, Karakiewicz PI, et al. Discrepancy between clinical and pathologic stage: impact on prognosis after radical cystectomy. Eur Urol 2007;51:137-49; discussion 149-51.

16. Szarvas T, Becker M, vom Dorp F, et al. Matrix metalloproteinase-7 as a marker of metastasis and predictor of poor survival in bladder cancer. Cancer Sci 2010;101:1300-8.

17. Jäger T, Tschirdewahn S, Vom Dorp F, et al. Siliconchiptechnology-based MMP-7 analysis in urine: an option for preoperative identification of lymph node metastasis in bladder cancer. Urologe A 2013;52:853-8.

18. Zhu C, Qi X, Zhou X, et al. Sulfatase 2 facilitates lymphangiogenesis in breast cancer by regulating

Cite this article as: Huang J, Li C, Zhang W, Yang F, Wang R, Zhang J, Li W, Yao X. SULF2 is a novel diagnostic and prognostic marker for high-grade bladder cancer with lymphatic metastasis. Ann Transl Med 2021;9(18):1439. doi: 10.21037/atm-21-4102
VEGF-D. Oncol Rep 2016;36:3161-71.

19. Lemjabbar-Alaoui H, van Zante A, Singer MS, et al. Sulf2, a heparan sulfate endosulfatase, promotes human lung carcinogenesis. Oncogene 2010;29:635-46.

20. Lai JP, Oseini AM, Moser CD, et al. The oncogenic effect of sulfatase 2 in human hepatocellular carcinoma is mediated in part by glypican 3-dependent Wnt activation. Hepatology 2010;52:1680-9.

21. Alhasan SF, Haugk B, Ogle LF, et al. Sulfatase-2: a prognostic biomarker and candidate therapeutic target in patients with pancreatic ductal adenocarcinoma. Br J Cancer 2016;115:797-804.

22. Yang H, Kim C, Kim MJ, et al. Soluble vascular endothelial growth factor receptor-3 suppresses lymphangiogenesis and lymphatic metastasis in bladder cancer. Mol Cancer 2011;10:36.

(English Language Editor: J. Jones) 